\title{
94. APORTACIONES A LA FLORA DE ANDALUCÍA. II.
}

\author{
Baltasar CABEZUDO, Andrés V. PÉREZ LATORRE, David NAVAS, \\ Gonzalo CABALLERO y Oscar GAVIRA
}

New data on the Andalusian flora. II.

Palabras clave. Juniperus, Dryopteris, Moehringia, Ulex, Frangula, Lithodora, Atropa, flora, corología, Andalucía, España.

Key words. Juniperus, Dryopteris, Moehringia, Ulex, Frangula, Lithodora, Atropa, flora, chorology, Andalusia, Spain.

Juniperus turbinata Guss.

MÁlAGA: Casares. Sierra de la Utrera. Calizas karstificadas. 10-2-2002. A. V. Pérez Latorre. MGC 48045.

La provincia de Málaga es probablemente la más diversa en cuanto a vegetación edafoxerófila gimnospérmica (Pérez Latorre et al., 1999); entre ella se encuentran los sabinares del género Juniperus (Pérez Latorre et al., 2001). Los sabinares caudados de Juniperus turbinata han estado tradicionalmente incluidos en sintaxones de medios litorales arenosos; sin embargo más recientemente se han ido describiendo situaciones ligadas a zonas de interior (Arista y Ortiz, 1995; Díez Garretas et al., 1996), pero se han separado sintaxonómicamente de sus "hermanas" litorales de Juniperion turbinatae, incluyéndolas en Asparago-Rhamnion oleoides (Rivas Martínez et al., 2002). En la provincia de Málaga, Juniperus turbinata está ligada con mayor preponderancia a paleoacantilados y paleoislas en las hoy interiores (Sanz de Galdeano y Vera, 1992) sierras de Huma, Cártama y Benahavís y en la localidad más recientemente hallada en Casares. La vegetación ibérica de Juniperus turbinata muestra por tanto dos grandes grupos ecológicamente y paleobotánicamente diferenciados: a/ los sabinares de paleoacantilados, probablemente supervivientes de finales del Terciario y por tanto de enorme valor paleobiogeográfico, que seguramente fueron origen de b/ los sabinares litorales sobre arenas, más recientes con toda seguridad debido a la histórica modificación y dinámica de las zonas de costa (Lario et al. 1993). Por tanto, creemos que ambos grupos de comunidades deben incluirse en Juniperion turbinatae, pero con esa diferenciación ecológica y paleobiogeográfica. La vegetación de esta nueva localidad malagueña se encuentra amenazada por actividades extractivas de áridos, estando la zona propuesta como LIC y como Monumento Natural de Andalucía (Torcal de la Utrera).

Dryopteris submontana (Fraser-Jenkins \& Jermy) Fraser-Jenkins

JAÉN. Albánchez de Úbeda. Parque Natural de Sierra Mágina. Sobre la Fuente del Espino. 1800 m. Grietas de grandes bloques calizos. 30SVG5976. 18-6-2002. B. Cabezudo, A. V. Pérez Latorre \& Y. Gil. MGC s/n.

Este pteridófito no había sido citado para la Sierra de Mágina por Cuatrecasas (1929), Salvo (1990) ni Ríos et al. (1993). Estos últimos autores habían confirmado su presencia en Jaén tras la primera cita de 1906 de Reverchon. La ecología es la clásica de $D$. submontana: grandes fisuras umbrosas entre bloques de rocas calizas, prácticamente ya en el horizonte inferior del piso bioclimático oromediterráneo (Dryopteridion submontanae, Thlaspietea), dominio climácico de los sabinar-enebrales de Pino-Juniperion sabinae.

Moheringia intrincata subsp. tejedensis (Huter, Porta \& Rigo ex Willk.) J. M. Monts.

Cuando ya Nieto (1987) o el propio libro rojo de la especies amenazadas de Andalucía en su capitulo exclusivamente dedicado $M$. tejedensis (taxon vulnerable) (Cabezudo et al., 2000) se da toda la información referente a esta subespecie en la provincia de Granada, (única localidad conocida: la cara norte de Sierra Tejeda) y habiéndose además estudiado más detalladamente su ecología y 
fitosociología por Mota et al. (1991), no se entiende la afirmación de Baena et al. (2003) sobre que "confirman... su presencia en Granada"(?).

\section{Ulex rivasgodayanus (Cubas) Cabezudo y Pérez} Latorre comb. et stat nov.

(U. parviflorus subsp. rivasgodayanus Cubas in Itinera Geobot. 15(2):709, 2002) (Basion.).

Se trata de la aulaga que ocupa las dolomías marmóreas fuertemente kakiritizadas fundamentalmente en la zona central del macizo de las Sierras Tejeda y Almijara (Málaga-Granada). En esa zona convive con U. parviflorus Pourr., pero éste ocupa las calizas y dolomías masivas o dolomías algo kakiritizadas pero edafizadas (con suelo intersticial) y domina en las zonas periféricas del complejo de Sierras, sobre todo al sur y noroeste. $U$. rivasgodayanus, por tanto, se encuentra en las provincias de Málaga y Granada, con sus mayores poblaciones entre los puertos de Cómpeta y Frigiliana, pero llegando hasta el extremo oeste de Sierra Tejeda, donde ya lo habían localizado Nieto et al. $(1988,1989)$, por lo que no se entienden las afirmaciones tanto de Cubas (Flora Iberica, 1999:234) citando Gr con interrogación, ni las de Baena et al. (2003) de que "su área se extiende hacia sierras limítrofes de la provincia de Granada (Sierra de Tejeda)...". U. rivasgodayanus también aparece sobre micaesquistos y cuarcitas en Sierra Tejeda, aunque deben existir hibridaciones con $U$. parviflorus, tal y como indica Cubas (1999) en los límites de sus poblaciones cuando contactan con las de U. parviflorus, encontrándose individuos intermedios entre ambas especies. Así mismo $U$. rivasgodayanus alcanza mayores altitudes de las indicadas en Flora Iberica (300-900 m.) subiendo por encima de los $1.200 \mathrm{~m}$ (MGC). Las citas de Mateos y Valdés (2003) y Talavera y Cubas (2002) para el Rif (sub. U. parviflorus subsp. rivasgodayanus) donde no existe $U$. parviflorus subsp. parviflorus (Talavera y Cubas, op. cit.), indican una mayor relevancia para el taxon, por su aislamiento y el mantenimiento de caracteres a tanta distancia geográfica. Incidiendo en la caracterización de $U$. rivasgodayanus, estudios sobre su especial fisiología y su adaptación a la regeneración rápida mediante semillas tras incendios recurrentes (Carreira et al., 1995) lo separan de $U$. parviflorus, que se regenera por rebrote tras el incendio. Por todo ello proponemos su cambio de status a especie, que sería por tanto un endemismo muy restringido de las Sierras de Tejeda y Almijara (S España) y Assifane, Taria y Targuist (N Marruecos).

\section{Frangula alnus Miller subsp. alnus}

GRANADA. Arenas del Rey. Parque Natural de las Sierras Tejeda, Almijara y Alhama. Río Cebollón. VF 28. 890 m. Dolomías. 24-6-2003. B. Cabezudo, G. Caballero, A. V. Pérez Latorre y D. Navas. MGC 55915.

GRANADA. Alhama de Granada. Parque Natural de las Sierras Tejeda, Almijara y Alhama. Barranco de las Tejadillas Oscuras. VF $28.1120 \mathrm{~m}$. Dolomías. 2-7-2003. B. Cabezudo, A. V. Pérez Latorre, G. Caballero y D. Navas. MGC 55917.

Especie no detectada hasta ahora en el complejo de las Sierras Tejeda-Almijara y que en Granada sólo es conocida de algunas localidades de Sierra Nevada (Erhn, 1968; Morales Abad, 1993), siendo muy escasa en toda Andalucía Oriental e inexistente al parecer en la Occidental, donde sí aparece $F$. alnus subsp. baetica que se extiende de modo aislado a Sierra Morena (Morales Abad, 1993). En el Parque Natural de Tejeda-Almijara-Alhama $F$. alnus habita en ríos permanentes de montaña con aguas básicas; concretamente es una especie constituyente de las saucedas blancas de Erico terminalis-Salicetum eleagni, bosques de ribera similares a los que ocupa en las Sierras de CazorlaSegura (Morales Abad, op. cit). Especies que le acompañan propias de las riberas son: Erica erigena, Salix atrocinerea, Salix eleagnos subsp. angustifolia y Populus nigra. También aparece en bosques caducifolios subriparios de Daphno-Aceretum granatense ligados a profundos cañones por donde discurren arroyos permanentes. En este caso las especies propias del bosque que acompañan a $F$. alnus son: Acer granatense, Sorbus aria, Amelanchier ovalis, Viburnum tinus, Arbutus unedo, Pistacia terebinthus y Lonicera splendida. Esta subespecie se podría considerar incluso menos abundante en Andalucía que la propia $F$. alnus subsp. baetica (especie vulnerable), por lo que podría ser designada para su protección.

Lithodora nitida (Ern.) R. Fernandes

MÁLAGA. Nerja. Parque Natural de las Sierras Tejeda, Almijara y Alhama. Barranco del Río Chíllar. VF $26.550 \mathrm{~m}$. Dolomías kakiritizadas. 20- 
3-2003. B. Cabezudo, A. V. Pérez Latorre, D. Navas y M. Becerra. MGC 55911.

GRANADA. Almuñecar. Sierra Almijara, Cerro de los Gigantes. VF 37.850 m. Dolomías. 165-2003. B. Cabezudo, G. Caballero y O. Gavira. MGC 55914.

Esta especie era conocida hasta ahora como endemismo de Jaén (Sierras Mágina y Pandera) y Córdoba (Sierra Horconera). Las poblaciones localizadas no solo se alejan mucho geográficamente de las anteriormente conocidas sino que además constituyen toda una novedad ecológica (bioclimática), fitocenológica y fenológica. L. nitida había sido considerada como una especie supraoromediterránea característica de los tomillares dolomitícolas de Andryalion agardhii (Convolvuletalia boissieri) por encima de $1400 \mathrm{~m}$ (Molero y Martínez, 1999) en el sector Subbético (provincia Bética). Las poblaciones almijarenses sin embargo se encuentran en el piso termomediterráneo, (la de Nerja 900 metros por debajo de las subbéticas), en pastizales glerícolas dolomitícolas de Andryalo-Crambion filiformis (Phagnalo-Rumicetalia) y en vegetación rupícola dolomitícola (Campanulion velutinae); secundariamente incluso entra en matorrales termófilos de Saturejo-Coridothymion. En todos los casos nos movemos dentro de los dominios de la serie edafoxerófilo-dolomitícola AlmijaroGranatense del boj (Buxus balearica). En el aspecto biológico es de reseñar que en la población nerjeña se han contabilizado 30 individuos en el núcleo principal y 1 en un hábitat secundario correspondiente a fisuras de paredones $(60$ individuos en la población granadina). La población malagueña muestra plántulas, al contrario que las de Jaén y Córdoba (Molero y Martínez, op. cit.) y una fenología también desviante como es lógico por la altitud, ya que florece en Marzo (final de AbrilMayo en las poblaciones subbéticas). En el aspecto ecomorfológico, los individuos de Nerja también son desviantes puesto que el tipo biológico es claramente el de un caméfito sufruticoso pulviniforme, mientras que las poblaciones de Jaén y Córdoba se ajustan a el de nanocaméfito escasamente ramoso con tallos postrado-ascendentes (Molero y Martínez, op. cit.). Igualmente las hojas muestran diferencias ya que mientras que las poblaciones subbéticas son de hasta $2 \times 0,8 \mathrm{~cm}$, las de Nerja las superan siendo hasta de $2,4 \times 0,9 \mathrm{~cm}$.
La existencia de estas poblaciones de una especie considerada en Peligro de Extinción (Junta de Andalucía), e incluida en el convenio de Berna, hacen de esta zona basal de Sierra Almijara una zona de gran importancia conservacionista respecto a flora, pues además cuenta con otras cuatro especies protegidas por la legislación andaluza como son Cneorum triccocum, Maytenus senegalensis, Buxus balearica y Anthyllis plumosa.

\section{Atropa baetica Willk.}

MÁlaGA. Alcaucín. Parque Natural de las Sierras Tejeda, Almijara y Alhama. Arroyo de los Pradillos. VF 08. 1550 m. Dolomías. 17-6-2003. A. V. Pérez Latorre y D. Navas. MGC 55913.

Hasta el momento no se conocían citas de esta especie en peligro de extinción (Herrera et al., 1999) en el complejo de las Sierras Tejeda-Almijara. La población localizada consta de un solo individuo con tres ramas y cuyas fenofases en el momento de su localización eran las de crecimiento vegetativo, floración y fructificación. La asociación vegetal que cobija a Atropa baetica en Sierra Tejeda es un juncal negro de Hyperico caprifolii-Schoenetum nigricantis (Molinio-Holoschoenion vulgaris), localizado en el borde de un arroyo que discurre sobre dolomías ligeramente kakiritizadas, y que muestra como especies más frecuentes a Schoenus nigricans, Cirsium sp., Linum narbonense, Potentilla reptans, Helleborus phoetidus, Digitalis obscura, Carex sp., Hypericum caprifolium y Berberis hispanica. Esta localidad podría ser "puente" entre las de la Serranía de Ronda y Antequera y las que podrían existir en Sierra Nevada (Herrera et al., op. cit.).

\section{BIBLIOGRAFÍA}

ARISTA, M. y P. L. ORTIZ -1995-Juniperus phoenicea subsp. turbinata en la Sierra de Grazalema. Acta Bot. Malacitana 20: 303-304.

BAENA, L., M. T. VIZOSO y F. B. NAVARRO 2003- Algunas novedades florísticas para la provincia de Granada. Lagascalia 23:157-162.

CABEZUDO, B., D. NAVAS, A. V. PÉREZ LATORRE, P. NAVAS, Y. GIL y T. NAVARRO -2000- Moehringia intricata subsp. tejedensis (Willk.) J. M. Monts. in: Blanca et al. (eds.) Libro Rojo de la Flora Silvestre Amenazada de 
Andalucía. Tomo II. Especies Vulnerables. Sevilla.

CARREIRA J. A., J. R. ARÉVALO y F. X. NIELL -1995-Soil degradation and nutrient availability in fire-prone mediterranean shrublands of southeastern Spain. Arid Soil Research and Rehabilitation 10: 55-56.

CUATRECASAS, J. -1929- Estudios sobre la flora y vegetación del macizo de Mágina. Trabajos Del Museo de Ciencias Naturales 12:3-510.

CUBAS, P. -1999- Ulex. L. in: Talavera et al. (eds.) Flora Iberica Vol VII(I). Real Jardín Botánico. CSIC. Madrid.

DÍEZ GARRETAS, B., A. ASENSI y V.E. MARTÍN OSORIO -1996- Comportamiento fitosociológico de Juniperus phoenicea L. s. I. en el sur de la Península Ibérica. Lazaroa 16: 159-167.

ERN, H. -1968- Über das vorkommen der birke (Betula L. spec.) in der Spanischen Sierra Nevada; Collect. Bot. (Barcelona) 7: 288-294.

HERRERA, C. M., E. HERNÁNDEZ BERMEJEO, P. LUQUE y A. BENAVENTE - 1999- Atropa baetica Willk. in Blanca et al. (eds.) Libro Rojo de la Flora Silvestre Amenazada de Andalucía. Tomo I. Especies en Peligro de Extinción. Sevilla.

LARIO, J., C. ZAZO, L. SOMOZA, J. L. GOY, M. HOYOS, P. G. SILVA y J. L. HERNÁNDEZ MOLINA -1993- Los episodios marinos cuaternarios de la costa de Málaga (España). Revista de la Sociedad Geológica de España 6:41-46.

MATEOS, M. A. y B. VALDÉS -2003- Nuevos taxones para el Rif Occidental. I. Lagascalia 23:133-156.

MOLERO, J. y M. J. MARTÍNEZ LIROLA -1999Lithodora nitida (Ern.) R. Fernandes in Blanca et al. (eds.) Libro Rojo de la Flora Silvestre Amenazada de Andalucía. Tomo I. Especies en Peligro de Extinción. Sevilla.

MORALES ABAD, M. J. -1993- Asientos para un Atlas Corológico de la Flora Occidental. Mapa 514; Fontqueria 36: 221-226.

MOTA, J. F., F. GÓMEZ MERCADO y F. VALLE -1991-Rupicolous vegetation of the betic ranges (south Spain). Vegetatio 94:101-113.

NIETO CALDERA, J. M. -1987- Estudio Fitocenológico de las Sierras Tejeda y Almijara. Tesis Doctoral. Universidad de Málaga.
NIETO CALDERA, J. M. y B. CABEZUDO - 1988Series de vegetación climatófilas de las Sierras Tejeda y Almijara. Acta Bot. Malacitana 13:229260.

NIETO CALDERA, J. M., B. CABEZUDO Y M. M. TRIGO PÉREZ -1989-. Series de vegetación edafófilas de las Sierras Tejeda y Almijara (Málaga, Granada, España). Acta Bot. Malacitana 14:161-170.

PÉREZ LATORRE, A. V., A. GALÁN DE MERA y B. CABEZUDO -1999- Propuesta de aproximación sintaxonómica sobre las comunidades de gimnospermas de la provincia Bética (España). Acta Bot. Malacitana 24: 257 262.

PÉrEZ LATORRE, A. V., B. CABEZUdo, A. GALÁN DE MERA y J. CARRIÓN -2001- El papel de las gimnospermas en la vegetación forestal de Andalucía. Medioambiente 38: 3033.

RÍOS, S., A. ROBLEDO \& F. ALCARAZ -1993Nuevas localidades de Dryopteris submontana (Fraser-Jenkins \& Jermy) Fraser-Jenkins en el macizo de Segura (S. E. de España). Acta Bot. Malacitana 18: 290.

RIVAS MARTÍNEZ, S., T. E. DÍAZ, F. FERNÁNDEZ GONZÁLEZ, J. IZCO, J. LOIDI y A. PENAS -2002-Vascular plant communities of Spain and Portugal. Itinera Geobotanica 15(1): 5-432.

SALVO TIERRA, E. - 1990- Guía de helechos de la Península Ibérica y Baleares. Ed. Pirámide.

SANZ DE GALDEANO, C. y J. A. VERA -1992Statigraphic record and paleographical context of the Neogene basins in the Betic Cordillera, Spain. Basin Research 4:21-36.

TALAVERA, S. y P. CUBAS -2002- Ulex L. in: Valdés et al. (eds.) Catalogue des plantes vasculaires du nord du Maroc, incluant des clés d'identification. Biblioteca de Ciencias. CSIC. Madrid.

Aceptado para su publicación en octubre de 2003

Dirección de los autores. Dpto. Biología Vegetal. Facultad de Ciencias. Universidad de Málaga. 29071, Málaga. 